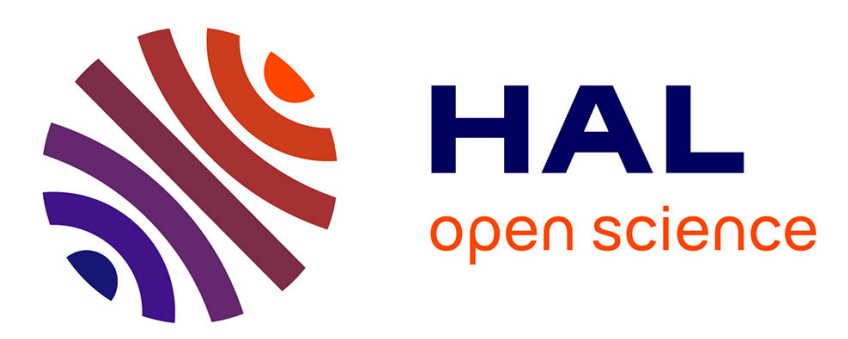

\title{
Direction-finding arrays of directional sensors for randomly located sources
}

Houcem Gazzah, Jean-Pierre Delmas, Sérgio M. Jesus

\section{To cite this version:}

Houcem Gazzah, Jean-Pierre Delmas, Sérgio M. Jesus. Direction-finding arrays of directional sensors for randomly located sources. IEEE Transactions on Aerospace and Electronic Systems, 2016, 52 (4), pp.1995 - 2003. 10.1109/TAES.2016.150655 . hal-01405774

\section{HAL Id: hal-01405774 \\ https://hal.science/hal-01405774}

Submitted on 30 Nov 2016

HAL is a multi-disciplinary open access archive for the deposit and dissemination of scientific research documents, whether they are published or not. The documents may come from teaching and research institutions in France or abroad, or from public or private research centers.
L'archive ouverte pluridisciplinaire HAL, est destinée au dépôt et à la diffusion de documents scientifiques de niveau recherche, publiés ou non, émanant des établissements d'enseignement et de recherche français ou étrangers, des laboratoires publics ou privés. 


\title{
Direction Finding Arrays of Directional Sensors for Randomly Located Sources
}

\author{
Houcem Gazzah, Jean Pierre Delmas and Sérgio M. Jesus
}

\begin{abstract}
The problem of directional sensor placement and orientation is considered when statistical information about the source direction-of-arrival is available. We focus on two-sensor arrays and form a Cramer-Rao-Bound based cost function that depends on the probability distribution of the coplanar source direction. Proper positioning and orientation of the sensors enable the two-sensor array to have an accuracy comparable to that of a 3 or 4 sensor uniform circular array.
\end{abstract}

Index Terms-Target localization, direction finding, directionof-arrival, directional sensors, Cramer-Rao lower bound (CRLB), geometry optimization.

\section{INTRODUCTION}

Direction-of-arrival (DOA) estimation is a major topic of antenna arrays signal processing, studied extensively over decades [1]. Source parameters (range, polarization, and, most notably, DOA) are extracted from the array manifold with an accuracy that depends on the estimation algorithm, but also on the array geometry. The potential of array geometry adaptation has been recently demonstrated [2], [3], [4], [5] to reduce the Cramer Rao Bound (CRB) on the DOA of deterministic/random far/near sources. For instance, (near) optimum nontrivial antenna array geometries were found that improve DOA estimation accuracy by $36 \%$ to $85 \%$, depending on the a priori information available about the source, compared to the more regularly used Uniform Circular Array (UCA) [4].

Similarly to previous work [2], [3], [4], [5], we continue to consider narrow-band sources. However, in this paper, sensors are not omni-directional, posing the problem of sensor orientations, in addition to sensor positions. We continue to refer to the CRB as our performance measure, both because it is algorithm-independent and achievable by a number of popular techniques [6], [7]. The CRB is different from a look direction to the other, so we use the Expected CRB (ECRB) to build a geometric cost function that also depends on the Probability Density Function (PDF) of the source DOA [2], [4], [8]. Optimization of the analytically intractable CRBbased cost function is achieved by means of a systematic search, preferably to heuristic techniques [2], [8], [9]. In order to reduce the computation burden, a minimal number of two sensors is considered. This is relevant to a number of applications that can accommodate only short aperture arrays,

Houcem Gazzah is with the Department of Electrical and Computer Engineering, University of Sharjah, 27272, UAE. E-mail: hgazzah@sharjah.ac.ae, Tel.: (971) 6.5050.917, Fax.: (971) 6.5050.872. Jean Pierre Delmas is with Telecom SudParis, Departement CITI, CNRS UMR 5157, Evry, France. Email: jean-pierre.delmas@it-sudparis.eu. Sérgio M. Jesus is with LARSyS, Universidade do Algarve, 8005-139 Faro, Portugal. Email: sjesus@ualg.pt. notably Autonomous Underwater Vehicles (AUV) used, e.g., in adaptive sampling networks [10], [11].

We focus on DOA estimation accuracy and, for instance, do not take array ambiguities into consideration. First, array ambiguities are less frequent when using arrays of directional sensors [12]. Second, they can be avoided by an appropriate choice of the spacing between adjacent sensors [12], which is allowed by the proposed algorithm. We, also, assume a source in the array plane. This is meaningful to a number of terrestrial applications [12], [13], [14] and amounts to prioritize the azimuth angle. With the azimuth as our unique parameterof-interest, we develop a scalar-valued performance measure and conduct an optimization in this perspective.

When the response of the directional sensors is not specified, the CRB has a non interpretable expression [12]. It is only once we assume a specific type of sensors, as in [12], [14], that performance analysis (and optimization) can be conducted. In our tests, we consider cardioid-type sensors for both the proposed geometry-optimized two-sensor array and the reference larger-sized UCAs. In the pessimistic case when there is no information about the source DOA, a scenario studied in [12], we find that sensors should be pointing at different directions, so that the CRB is finite at every possible look direction and the subsequent ECRB is finite as well. If the source DOA is known with (moderate) uncertainty, the optimized two-sensor array has a better accuracy than the three-sensor UCA. The fact that we can achieve with two sensors an accuracy normally achievable by (a UCA of) three sensors implies significant reduction of the size, weight, power and cost of the system [12], since every single sensor requires a separate receiver channel.

The paper is organized as follows. In Sec. II, we introduce the observation model and develop expressions of the CRB. In Sec. III, the CRB of the array of two directional sensors is studied in detail and a subsequent array geometry procedure is defined. In Sec. IV, tests are conducted using cardioid-type sensors to compare the optimized array to larger-sized UCAs. Finally, a conclusion is given in Sec. V.

\section{Signal Model And General Results}

A narrow-band source is emitting a signal $s(t)$ of wavelength $\lambda$ in the direction of an array of $M$ co-planar sensors. In the $[O, x, y)$ plane, sensor $m$ is placed at point $P_{m}$ with a distance $O P_{m}=\rho_{m} \lambda$ from the origin $O$ and an angle $\phi_{m}$ between the $[O, x)$ axis and $\left[O, P_{m}\right)$. The far-field source is seen at the antenna array under the DOA angle $\theta$, restricted to be in $[-\pi, \pi]$, w.r.t the $[O, x)$ axis. All angles are measured 
counter-clockwise. The array output at time index $t$

$$
\mathbf{x}(t)=\mathbf{a}(\theta) s(t)+\mathbf{n}(t), t=t_{1}, \ldots, t_{N}
$$

is a scaled and noise-corrupted replica of the DOA-dependent Array Response Vector (ARV) a $(\theta)$. The ARV is an extension of the array steering vector that incorporates gains of the sensors [15]. Its $m$-th component is given by

$$
[\mathbf{a}(\theta)]_{m}=g_{m}(\theta) \exp \left[j 2 \pi \rho_{m} \cos \left(\theta-\phi_{m}\right)\right],
$$

where we have assumed that sensor $m$, not necessarily omnidirectional, has a directional response described by the function $g_{m}(\theta)^{1}$.

Snapshots $(\mathbf{x}(t))_{t=t_{1}, \ldots, t_{N}}$ are used to estimate the parameter $\theta$ using a variety of techniques. The CRB [16] often serves as a benchmark to compare estimation performance of the different estimation algorithms. It represents the lowest mean square error achievable by any unbiased estimator. The CRB is also of practical importance [13] because (in the single source case considered here) is achieved (asymptotically, as the number of snapshots increases) by both the high-resolution MUSIC algorithm [6] and the low-resolution beam-forming techniques [7]. The following statistical properties are often assumed about $s(t)$ and $\mathbf{n}(t)$ :

(i) $s(t)$ and $\mathbf{n}(t)$ are independent,

(ii) $(\mathbf{n}(t))_{t=t_{1}, \ldots, t_{N}}$ are independent, zero-mean circular Gaussian distributed with covariance $\mathrm{E}\left[\mathbf{n}(t) \mathbf{n}^{H}(t)\right]=\sigma_{n}^{2} \mathbf{I}, \mathbf{I}$ being the $M \times M$ identity matrix,

(iii) $(s(t))_{t=t_{1}, \ldots, t_{N}}$ are assumed to be either deterministic unknown parameters (the so-called conditional or deterministic model), or independent zero-mean circular Gaussian distributed with variance $\sigma_{s}^{2}$ (the so-called unconditional or stochastic model). The above conditions, while of common use in performance analysis (see e.g., [16]), do not account for some practical aspects (spatially/temporally correlated noise, mutual coupling, ...) whose impact is to be evaluated empirically, rather than analytically, which is beyond the scope of this study. The CRBs associated with both models have been proved in [5] to be proportional (one to the other) ${ }^{2}$. For instance, the CRB associated with the first model is given by

$$
\operatorname{CRB}(\theta)=\frac{\sigma_{n}^{2}}{2 N \sigma_{s}^{2}} F^{-1}(\theta),
$$

where, given $\mathbf{a}^{\prime}(\theta) \hat{=} d \mathbf{a}(\theta) / d \theta$, the scalar-valued

$$
F(\theta)=\left\|\mathbf{a}^{\prime}(\theta)\right\|^{2}-\frac{\left|\mathbf{a}^{H}(\theta) \mathbf{a}^{\prime}(\theta)\right|^{2}}{\|\mathbf{a}(\theta)\|^{2}}
$$

is a convenient design criterion because it is independent from the noise/signal power and the number of snapshots. Consequently, we will be referring to the above expressions throughout the paper.

\footnotetext{
${ }^{1}$ Similarly as in [12], the sensor response $g_{m}(\theta)$ is a voltage or current gain, different from the sensor power response $g_{m}^{2}(\theta)$.

${ }^{2}$ They are equal if $\|\mathbf{a}(\theta)\|^{2} \sigma_{s}^{2} \gg \sigma_{n}^{2}$.
}

\section{Theoretical Development}

\section{A. Optimization Criterion}

The array is made of two directional sensors [17]. One is placed at the origin, while the position of the other one, characterized by distance $\rho \hat{=} \rho_{2}$ and angle $\phi=\phi_{2}$, is to be determined, along with the orientation of each sensor. Given the following expression of the ARV

$$
\mathbf{a}(\theta)=\left[g_{1}(\theta), g_{2}(\theta) \exp [j 2 \pi \rho \cos (\theta-\phi)]\right]^{T},
$$

we prove in Appendix A that

$$
F(\theta)=g_{1}^{2}(\theta) \frac{\left[h^{\prime}(\theta)\right]^{2}+4 \pi^{2} \rho^{2} h^{2}(\theta) \sin ^{2}(\theta-\phi)}{1+h^{2}(\theta)},
$$

where $h(\theta) \hat{=} g_{2}(\theta) / g_{1}(\theta)$, assuming none of the sensors has a strictly zero gain at any direction. Here, $h^{\prime}(\theta)$ can be interpreted as a measure of the mismatch between the two sensors' directivity patterns. Based on (5), we can make the following two remarks:

(i) If the two sensors are identical and pointing in the same direction, $g(\theta)=g_{1}(\theta)=g_{2}(\theta)$, then $F(\theta)=$ $2 \pi^{2} g^{2}(\theta) \rho^{2} \sin ^{2}(\theta-\phi)$ is zero when the source is at the array endfire direction (i.e, $\theta=\phi$ ), regardless of how the sensors are directed.

(ii) In contrast, if the two sensors have different directivity patterns, $F(\theta)$ can be made arbitrarily high provided $h^{\prime}(\theta)$ is large enough, including for $\theta=\phi$. In other words, sources that are in the array endfire direction can be precisely identified only if we use different and/or differently oriented sensors.

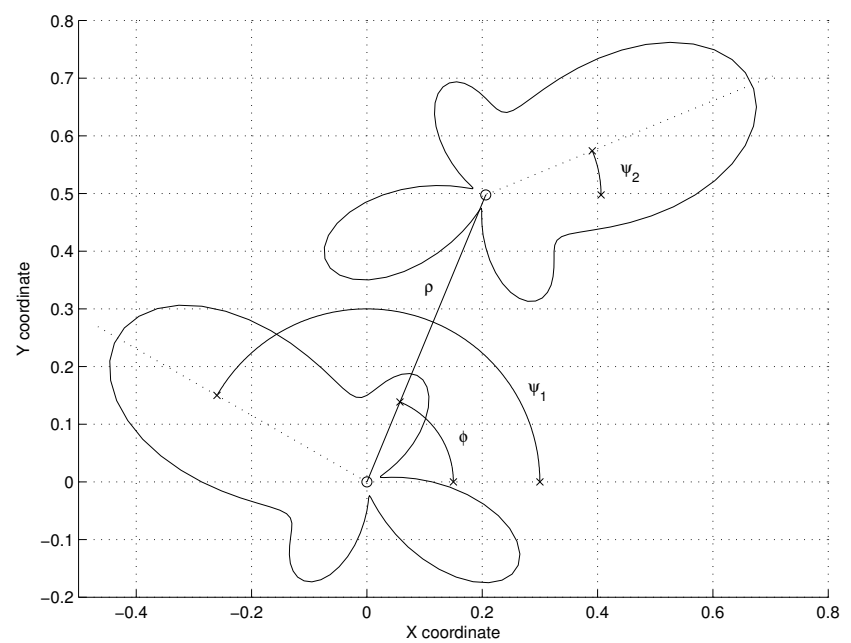

Fig. 1. Positions and orientations of the two directional sensors for an arbitrarily-shaped sensor response $g(\theta)$. The lines show individual responses for each sensor.

In practice, we are likely to use identical sensors pointing at different directions, i.e.

$$
g_{m}(\theta) \hat{=} g\left(\theta-\psi_{m}\right), m=1,2 .
$$

As illustrated in Fig. 1, the array configuration is, now, parameterized by geometrical parameters $\rho, \phi, \psi_{1}$ and $\psi_{2}$, in 
function of which $F(\theta)$ is expressed, as follows

$$
F(\theta)=\frac{\left[\frac{g^{\prime}\left(\theta-\psi_{1}\right)}{g\left(\theta-\psi_{1}\right)}-\frac{g^{\prime}\left(\theta-\psi_{2}\right)}{g\left(\theta-\psi_{2}\right)}\right]^{2}+4 \pi^{2} \rho^{2} \sin ^{2}(\theta-\phi)}{\frac{1}{g^{2}\left(\theta-\psi_{1}\right)}+\frac{1}{g^{2}\left(\theta-\psi_{2}\right)}}
$$

This function is to be interpreted as the ability of the antenna array to accurately localize a source with the specific DOA $\theta$. Since the source DOA cannot be (exactly) known in advance, the overall array performance is more suitably measured in terms of the so-called expected CRB [2], [4], [8] defined as $E C R B \hat{=} \mathrm{E}[C R B(\theta)]$. By adopting the ECRB as a performance criterion, we, implicitly, allow the CRB to be high at directions where the source is less likely to show up. The $a$ priori information about the source DOA is available in the form of a PDF $f(\theta)$, leading to

$$
\mathrm{ECRB}=\frac{\sigma_{n}^{2}}{2 N \sigma_{s}^{2}} \int_{-\pi}^{\pi} \frac{f(\theta)}{F(\theta)} d \theta .
$$

Minimizing the ECRB for fixed powers $\sigma_{n}^{2}$ and $\sigma_{s}^{2}$ and number $N$ of snapshots is tantamount to minimizing

$$
\frac{2 N \sigma_{s}^{2}}{\sigma_{n}^{2}} \mathrm{ECRB}=\int_{-\pi}^{\pi} \frac{f(\theta)}{F(\theta)} d \theta .
$$

\section{B. Optimization Procedure}

Inter-sensor spacing $\rho$ is assumed to be fixed based on considerations other than estimation accuracy (e.g. coupling and ambiguity considerations), independently from $\psi_{1}, \psi_{2}$ and $\phi$, which remain to be determined by minimization of the above ECRB criterion. This is to be achieved by means of a 3D systematic search. It will be possible to reduce the search area thanks to some properties of the cost function. In fact, (6) is unchanged if i) $\phi$ is replaced by $\phi+\pi$; or ii) $\left(\psi_{1}, \psi_{2}\right)$ is replaced by $\left(\psi_{2}, \psi_{1}\right)$. Consequently, the systematic search can be restricted to $\phi$ in $[-\pi / 2, \pi / 2], \psi_{1}$ in $[-\pi, \pi], \psi_{2}$ in $[-\pi, \pi]$ and $\psi_{1} \leq \psi_{2}$. Notice that, for the sake of numerical stability, configurations where $\psi_{1}=\psi_{2}$ are not tested because, then, the function $1 / F(\theta)$ is divergent (at $\theta=\phi$ ), and so is the ECRB.

Further simplification is possible if both the sensor response and the DOA PDF are even, i.e. respectively $g(-\theta)=g(\theta)$ and $f(-\theta)=f(\theta)$. Under these assumptions, we have

$$
\begin{aligned}
& \frac{2 N \sigma_{s}^{2}}{\sigma_{n}^{2}} \mathrm{ECRB} \\
= & \int_{0}^{\pi}\left\{\frac{\frac{1}{g^{2}\left(\theta-\psi_{1}\right)}+\frac{1}{g^{2}\left(\theta-\psi_{2}\right)}}{\left[\frac{g^{\prime}\left(\theta-\psi_{1}\right)}{g\left(\theta-\psi_{1}\right)}-\frac{g^{\prime}\left(\theta-\psi_{2}\right)}{g\left(\theta-\psi_{2}\right)}\right]^{2}+4 \pi^{2} \rho^{2} \sin ^{2}(\theta-\phi)}\right. \\
+ & \left.\frac{\frac{1}{g^{2}\left(\theta+\psi_{1}\right)}+\frac{1}{g^{2}\left(\theta+\psi_{2}\right)}}{\left[\frac{g^{\prime}\left(\theta+\psi_{1}\right)}{g\left(\theta+\psi_{1}\right)}-\frac{g^{\prime}\left(\theta+\psi_{2}\right)}{g\left(\theta+\psi_{2}\right)}\right]^{2}+4 \pi^{2} \rho^{2} \sin ^{2}(\theta+\phi)}\right\} f(\theta) d \theta,
\end{aligned}
$$

so that the ECRB is unchanged if $\psi_{1}, \psi_{2}$ and $\phi$ are replaced by $-\psi_{1},-\psi_{2}$ and $-\phi$, respectively. It follows that, for such a case, we can further restrict $\phi$ to be in $[0, \pi / 2]$.

\section{OPTIMIZATION RESUlts}

The proposed array (to which we refer as $\mathrm{CAM}^{3}$ ) is compared to larger-sized UCA arrays. In all examples, half-awavelength inter-sensor spacing is assumed, in order to avoid (first-order) array ambiguities [18], [19]. The optimization problem depends on (i) the type of sensors (assumed in Sec. IV-B to be of cardioid-type) and (ii) the distribution of the source azimuth angle (assumed in Sec. IV-C and Sec. IV-D to be uniform and normal to describe worst case and realistic scenarios, respectively). The resolution of the systematic search grid is set to 2 [DEG]. We, first, start by presenting some results about the reference UCA.

\section{A. The reference $U C A$}

We test our geometry-optimized two-sensor array simultaneously with the commonly used UCA, made of $M=3,4,5, \cdots$ directional sensors. For the UCA, sensors are placed uniformly along the circle, i.e. at angles $\phi_{m}=2 \pi(m-1) / M, m=$ $1, \cdots, M$. The circle radius is $R \lambda$ where $R=\rho /[2 \sin (\pi / M)]$ ensures an inter-sensor spacing equal to $\rho$. As pointed out in [12], one can avoid array ambiguities in a UCA by appropriately choosing the inter-sensor spacing $\rho$. The directional sensors are pointed in the same direction as the sensors, i.e. $g_{m}(\theta)=g\left(\theta-\phi_{m}\right)$, a fixed-geometry design previously proposed in [12], [14], [20]. The UCA geometry is special in that it verifies, for all $k$ not multiple of $M$,

$$
\sum_{m=1}^{M} \exp \left(k \phi_{m}\right)=0
$$

which will be useful to obtain the compact CRB expressions (10) and (13)-(16).

Isotropy is a desired feature of antenna arrays that is fulfilled by UCAs when they are composed of omni-directional sensors [3]. Interestingly enough, we prove that the UCA isotropy may be preserved even when the constituent sensors are not isotropic. We focus our attention on sensors with arbitrary but symmetrical (even) pattern $g(\theta)$, which are widely encountered in practice. For such sensors, we can write

$$
g(\theta)=g_{0}\left[1+\sum_{k=1}^{K} \beta_{k} \cos (k \theta)\right]
$$

where $\left(\beta_{k}\right)_{k=1, \ldots, K}$ satisfy $1+\sum_{k=1}^{K} \beta_{k} \cos (k \theta) \geq 0$ for all $\theta$ and, also, $\beta_{1} \geq 0, \ldots, \beta_{K-1} \geq 0, \beta_{K}>0$, hence ensuring a maximum gain in the (zero degrees) look direction. Coefficients $\beta_{k}, k=1, \cdots, K$, can be easily computed by means of a (truncated) Fourier cosine expansion of $g(\theta)$ whether $g(\theta)$ is available in analytical or numerical form.

We prove in Appendix B the following result: If the directional sensor has a symmetric response $g(\theta)$ as in (9), then the UCA made of $M$ such sensors is isotropic if $M>2(K+1)$,

\footnotetext{
${ }^{3}$ So-named in reference to the chameleon whose eyes can rotate and move independently from each other.
} 
and, then, it verifies

$$
\begin{gathered}
F(\theta)=\frac{M g_{0}^{2}}{2}\left[\sum_{k=1}^{K} k^{2} \beta_{k}^{2}+\pi^{2} R^{2}\left(4+\beta_{1}^{2}-4 \beta_{2} \delta_{K>1}\right.\right. \\
\left.\left.+2\left(\sum_{k=2}^{K} \beta_{k}^{2}\right) \delta_{K>1}-2\left(\sum_{k=1}^{K-2} \beta_{k} \beta_{k+2}\right) \delta_{K>2}\right)\right],(10)
\end{gathered}
$$

where $\delta_{A}=1$ if condition $A$ is satisfied and 0 , otherwise.

Before we interpret this result, we first mention that there is no direct relationship between directivities of the sensors (defined as $D \hat{=}\left[\max _{\theta} g^{2}(\theta)\right] /\left[\frac{1}{2 \pi} \int_{-\pi}^{\pi} g^{2}(\theta) d \theta\right]$ ) and isotropy of the UCA, except for specific families of patterns. For example, let's consider sensors from [12] with response $g(\theta)=g_{0}[1+\cos (\theta)]^{K}$, whose directivity, proved in Sec. C to be equal to

$$
D=2^{4 K} / \sum_{\ell=0}^{K} \frac{(2 K) ! 2^{2(K-\ell)}}{(\ell !)^{2}(2(K-\ell)) !}
$$

increases with $K(D=1,2.66,3.66,4.43$ and 5.68 for $K=0,1,2,3$ and 4 , respectively). By application of (10), a minimum of $1+2(K+1)$ such sensors is needed to make the so-composed UCA an isotropic one. Result (10) contrasts with the UCA of omni-directional sensors that is isotropic if $M>2$ [21]. It proves that a UCA with directional sensors (regardless of how much directional they are) can still be isotropic if the number of sensors is sufficiently large.

Of special interest are cardioid sensors of frequent use in acoustic systems [22]. They are characterized by a directional response of the form [23]

$$
g(\theta)=g_{0}[1+\beta \cos (\theta)]
$$

parameterized by constants $g_{0}$ and $\beta$. Application of (10) implies that the UCA is isotropic if populated with 5 or more such sensors. Then, it verifies

$$
F(\theta)=\frac{M g_{0}^{2}}{2}\left[\beta^{2}+\pi^{2} R^{2}\left(4+\beta^{2}\right)\right],
$$

consistently with [21] for omni-directional sensors $(\beta=0)$. For completeness, in order to also address non-isotropic UCA of cardioid sensors, we prove in Appendix D the following expressions for arbitrarily sized UCA of cardioid sensors

$$
\begin{aligned}
\frac{2 F(\theta)}{g_{0}^{2}}= & 4 \sin ^{2}(\theta)\left\{\beta^{2}+\cos ^{2}(\theta)\left[\pi^{2} \rho^{2}\left(4+\beta^{2}\right)\right.\right. \\
& \left.\left.-\beta^{2} \frac{\beta^{2}+4 \pi^{2} \rho^{2}}{1+\beta^{2} \cos ^{2}(\theta)}\right]\right\}, M=2 \\
= & \pi^{2} \rho^{2}\left[4+\beta^{2}-4 \beta \cos (3 \theta)-\frac{\beta^{4} \sin ^{2}(3 \theta)}{2+\beta^{2}}\right] \\
& +3 \beta^{2}, M=3 \\
= & 4 \beta^{2}+4 \pi^{2} \rho^{2}\left[2+\beta^{2} \sin ^{2}(2 \theta)\right], M=4 \\
= & M\left[\beta^{2}+\pi^{2} \rho^{2} \frac{1+\frac{\beta^{2}}{4}}{\sin ^{2}\left(\frac{\pi}{M}\right)}\right], M>4
\end{aligned}
$$

\section{B. Sensors}

In our tests, we consider cardioid-type sensors as defined in (12), parameterized by constants $g_{0}$ and, more importantly, $\beta$ that controls the sensor directivity $D$ found to be equal to $(1+\beta)^{2} /\left[1+\beta^{2} / 2\right]$, which increases from 0 to 2.66 when $\beta$ increases from 0 to 1 . Substituting (12) into (6) leads to the following update of $F(\theta)$

$$
\begin{aligned}
& \frac{F(\theta)}{g_{0}^{2}}= \\
& \frac{\beta^{2}\left[\frac{\sin \left(\theta-\psi_{1}\right)}{1+\beta \cos \left(\theta-\psi_{1}\right)}-\frac{\sin \left(\theta-\psi_{2}\right)}{1+\beta \cos \left(\theta-\psi_{2}\right)}\right]^{2}+4 \pi^{2} \rho^{2} \sin ^{2}(\theta-\phi)}{\frac{1}{\left[1+\beta \cos \left(\theta-\psi_{1}\right)\right]^{2}}+\frac{1}{\left[1+\beta \cos \left(\theta-\psi_{2}\right)\right]^{2}}}
\end{aligned}
$$

where the right-hand side, advantageously, depends on $\beta$ only, as long as the sensor is concerned. Hence, we adapt the initial criterion (7) to minimize, instead, $\frac{2 g_{0}^{2} N \sigma_{s}^{2}}{\sigma_{n}^{2}}$ ECRB given by the $\beta$-dependent

$$
\begin{aligned}
& C \hat{=} \int_{-\pi}^{\pi} \\
& \frac{\left(\frac{1}{\left[1+\beta \cos \left(\theta-\psi_{1}\right)\right]^{2}}+\frac{1}{\left[1+\beta \cos \left(\theta-\psi_{2}\right)\right]^{2}}\right) f(\theta)}{\beta^{2}\left[\frac{\sin \left(\theta-\psi_{1}\right)}{1+\beta \cos \left(\theta-\psi_{1}\right)}-\frac{\sin \left(\theta-\psi_{2}\right)}{1+\beta \cos \left(\theta-\psi_{2}\right)}\right]^{2}+4 \pi^{2} \rho^{2} \sin ^{2}(\theta-\phi)} d \theta .
\end{aligned}
$$

\section{No a priori}

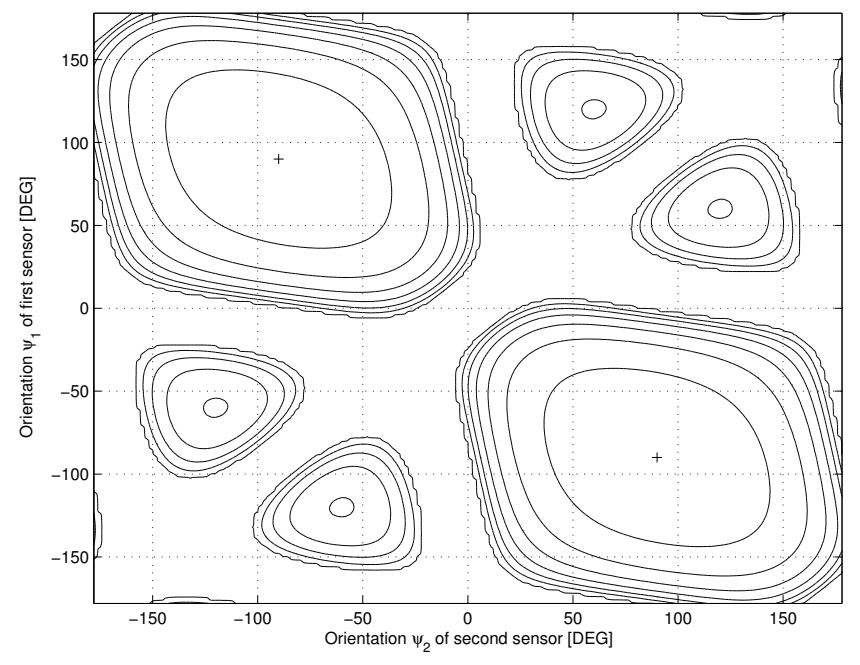

Fig. 2. The cost function $C$ for $\beta=0.8$. Global minima are shown as ' + ' dots.

We consider the case of a source DOA uniformly distributed over $[-\pi, \pi]$. There is, actually, an infinity of equivalent solutions. In fact, because $1 / F(\theta)$ is being integrated over one period, it can be shown that $C$ is unchanged by a translation of $\phi$. Hence, we assume $\phi=0$ within this section. A sample (for $\beta=0.8$ ) cost function $C$ is presented in Fig. 2, showing optimality is met at $\psi_{1}=-\psi_{2}=\pi / 2$, which is verified for all possible values of $\beta$. In general, optimality is met with the two sensors pointing in opposite directions, orthogonally to the axis linking the two sensors. This axis, however, can be randomly oriented. We denote as CAMU the two-sensor array depicted in Fig. 3 and characterized by $\phi=0, \psi_{1}=\pi / 2$ 


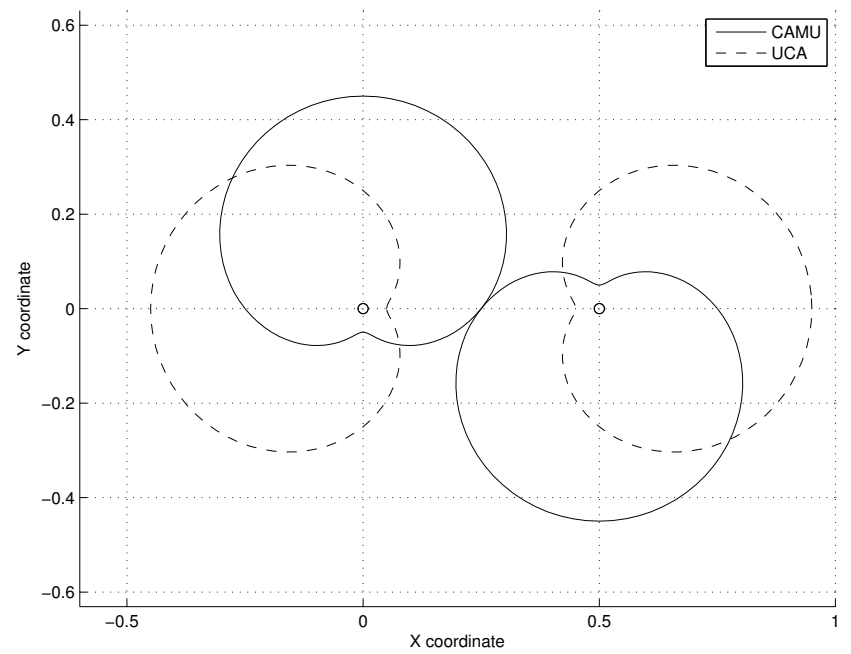

Fig. 3. Sensor orientations of CAMU and UCA2 arrays in the $(x, y)$ plane. Sensors positions, shown as circular dots, are the same for both arrays. The lines show individual responses for each sensor when $\beta=0.8$.

and $\psi_{2}=-\pi / 2$. It is optimal for a source with a uniformly distributed DOA.

Contrarily to the two-sensors UCA, the CAMU array does not have an infinite CRB at any direction, as is clear from Fig. 4. As a consequence, its accuracy (in terms of the ECRB) is finite, of the same order as that of the three-sensor UCA. Also, Fig. 4(a) shows that, as $\beta$ of the constituent sensors increases, the CRB is reduced in the endfire direction and is increased at broadside. A good compromise seems to be attained for $\beta$ around 0.5, where the CRB fluctuates the least and the CAMU is closest to be isotropic. This would be the best design for those applications requiring (more or less) the same accuracy at all possible look directions.

As can be concluded from Fig. 5, the use of directional sensors is more beneficial to CAMU than to UCA. With this particular configuration of the CAMU array, substituting $\phi=$ $0, \psi_{1}=\pi / 2$ and $\psi_{2}=-\pi / 2$ into (17) results into

$$
\frac{F(\theta)}{2 g_{0}^{2}}=\frac{\beta^{2} \cos ^{2}(\theta)}{1+\beta^{2} \sin ^{2}(\theta)}+\pi^{2} \rho^{2} \sin ^{2}(\theta) \frac{\left[1-\beta^{2} \sin ^{2}(\theta)\right]^{2}}{1+\beta^{2} \sin ^{2}(\theta)},
$$

which is not zero in any direction, as long as $\beta$ is not zero. Again, from Fig. 5, best performance is obtained using sensors with $\beta$ slightly larger than $1 / 2$. To be concluded from Fig. 5 , the optimally-configured two-sensor array is outperformed by the larger three-sensor UCA. However, this is true only because, disadvantageously, this PDF expresses no a priori about the source DOA. As shown in the next section, the situation is more profitable to our design if (more) information is available about the source DOA.

\section{Normal a priori}

In some realistic scenarios, the source DOA is expected to appear in a given direction $\Omega$ assumed, without loss of generality, to be 0 . The DOA is modeled as a centered normal random variable and the optimal two-sensor array is

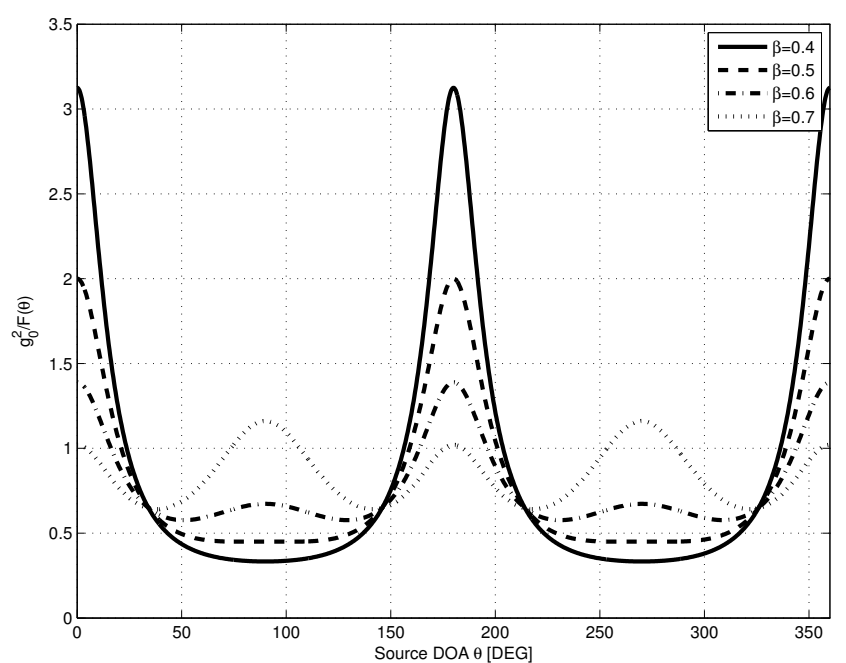

(a)

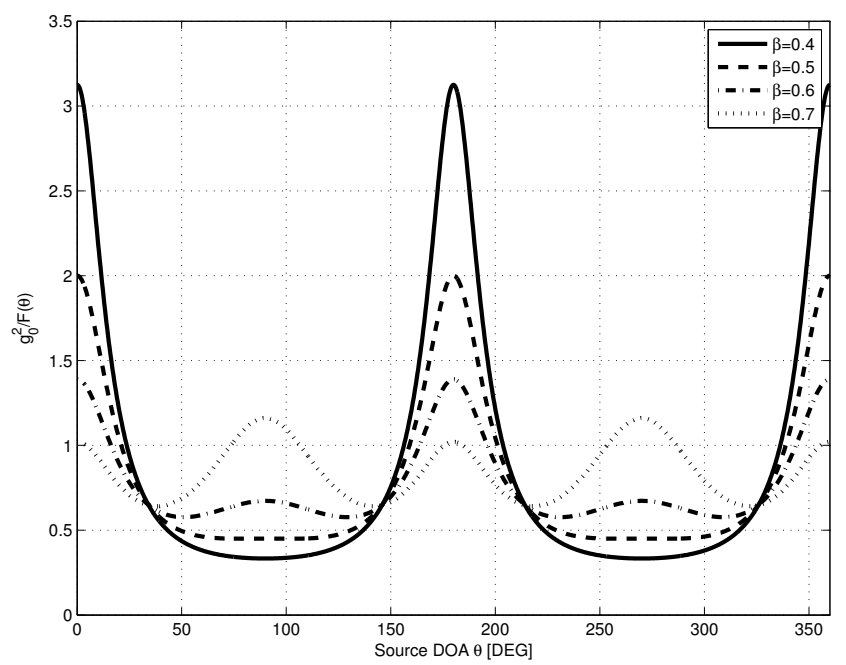

(b)

Fig. 4. $g_{0}^{2} / F(\theta)$ (which is proportional to the CRB), for all possible source DOAs, for both the CAMU array, in (a) and (b), and the reference UCAs in (b). In (b), sensors are such that $\beta=1 / 2$.

studied as function of the standard deviation $\sigma$. Geometry and performance of the optimal CAM array are shown in Fig. 6 for $\sigma$ not exceeding 40 [DEG] in order to ensure that the PDF $f(\theta)=\frac{1}{\sqrt{2 \pi} \sigma} \exp \left[-\theta^{2} /\left(2 \sigma^{2}\right)\right]$ is almost zero for any $\theta$ not in $[-\pi, \pi]$. Results shown in Fig. 6(a) suggest that there is a range of $\sigma$ where the optimized two-sensor array performs closely to (better and worse than) the three-sensor UCA. In this case, the two sensors of the optimized array are placed orthogonally ( $\phi=\pi / 2)$ to the expected source DOA and are pointing into symmetric (w.r.t. DOA) directions $\left(\psi_{1}=-\psi_{2}\right)$. The larger the uncertainty $\sigma$ about $\Omega$, the larger the offset $\left|\psi_{1}\right|=\left|\psi_{2}\right|$, as shown in Fig. 6(b). However, for an excessively large $\sigma$ (i.e. limited a priori information), geometry optimization is less beneficial and performance is not much better than that of the 


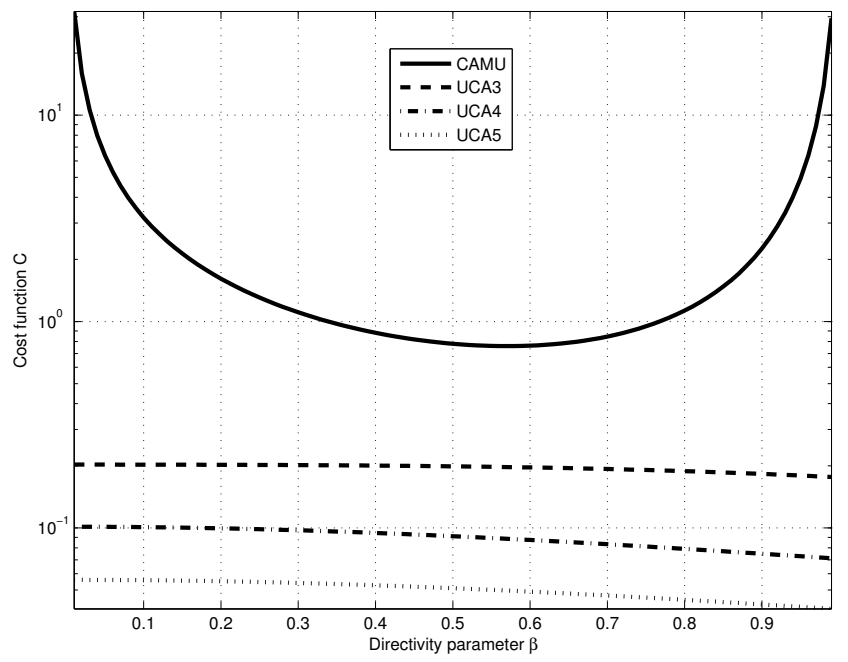

Fig. 5. Compared performance of CAMU and UCA in terms of the cost function $C$ (proportional to the ECRB) as function of $\beta$ (expressing the directivity of the cardioid sensor), for a uniformly distributed source DOA.

\section{CAMU array.}

\section{E. Arbitrary a priori}

A more general PDF model is that of a mixture of Gaussian distributions with different means (that express the different look directions) and variances (that express the uncertainty about the look directions). Strictly speaking, we let $P$ be the number of look directions. We let $\kappa_{p}, \Omega_{p}$ and $\sigma_{p}$ be the weight, the mean and the standard deviation relative to the $p$-th distribution, so that

$$
f(\theta)=\sum_{p=1}^{P} \frac{\kappa_{p}}{\sigma_{p} \sqrt{2 \pi}} \exp \left[-\frac{\left(\theta-\Omega_{p}\right)^{2}}{2 \sigma_{p}^{2}}\right],
$$

for any $\theta$ in $[-\pi, \pi]$, where $\sum_{p=1}^{P} \kappa_{p}=1$. We assume $-\pi<\Omega_{p}-3 \sigma_{p}$ and $\Omega_{p}+3 \sigma_{p}<\pi$ for all $p$ in order to have $\sum_{p=1}^{P} \frac{\kappa_{p}}{\sigma_{p} \sqrt{2 \pi}} \exp \left[-\left(\theta-\Omega_{p}\right)^{2} /\left(2 \sigma_{p}^{2}\right)\right] \simeq 0$ for any $\theta$ not in $[-\pi, \pi]$. In the simulations, we have assumed equally likely look directions, i.e. $\kappa_{1}=\cdots=\kappa_{P}=1 / P$ and the same uncertainty $\sigma_{1}=\cdots=\sigma_{P}=10$ [DEG].

In a first set of simulations, and in order to explore the potential of the proposed optimized array for arbitrary PDFs, we assume two possible look directions $\Omega_{1}$ and $\Omega_{2}$. Without loss of generality (and in order to obtain an even PDF), we choose $\Omega_{1}=-\Omega_{2}$ ranging from 10 to 90 [DEG]. As illustrated in Fig. 7, we compare the performance of the optimized twosensor array (CAM) to those of the non-optimized arrays (the two-sensor CAMU and UCAs of 3,4 and 5 sensors). We realize that, overall, the optimized two-sensors array performs closely to the 3 -sensor UCA. The CAMU array, who has a minimum size and a non-adaptive geometry, is distinctively the one with the lowest performance.

To illustrate a more irregular PDF, we consider the example where $\Omega_{1}=20, \Omega_{2}=50$ and $\Omega_{3}=80$ [DEG]. If sensors

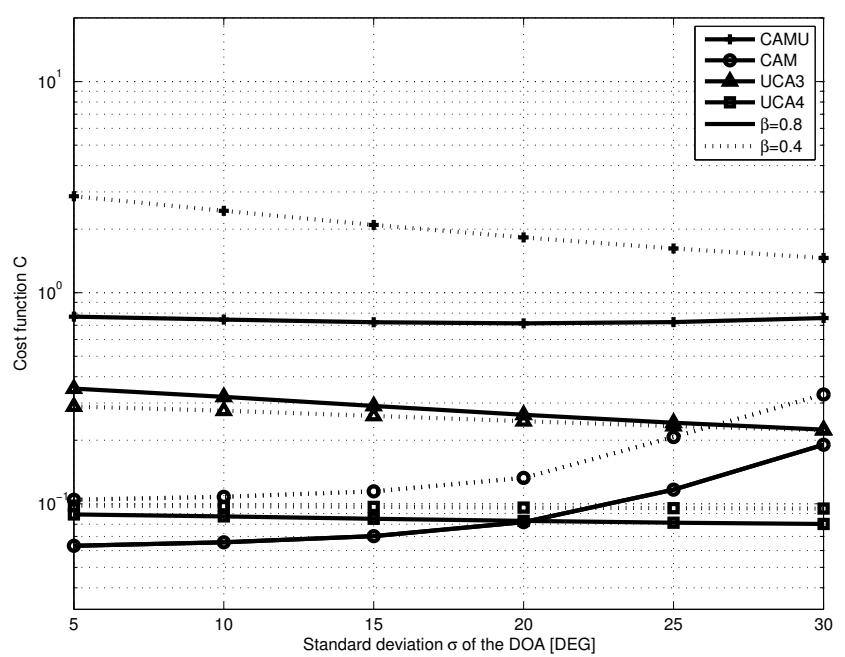

(a) Performance of the optimized CAM array

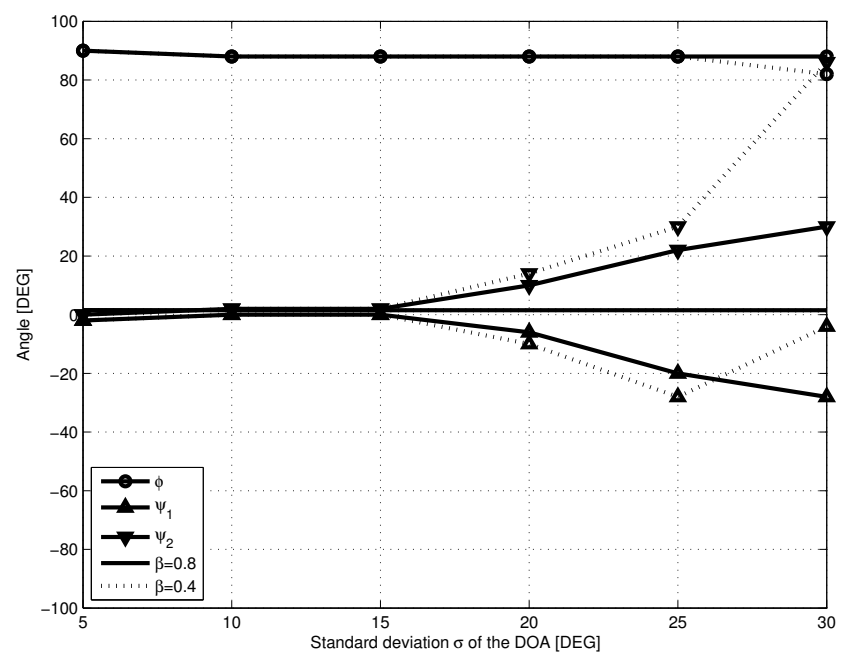

(b) Shape of the optimized CAM array

Fig. 6. Performance (a) and shape (b) of the optimized CAM array, comparatively to non-adaptive fixed-geometry CAMU and UCA arrays, for a zero-mean normally distributed source DOA with a standard deviation as shown along the horizontal axis.

with $\beta=0.8$ are to be used, then it is found that the sodistributed source is best localized using the optimized twosensor array characterized by $\phi=-40, \psi_{1}=48$ and $\psi_{2}=$ 50 [DEG]. Such array achieves a performance, in terms of $C$, equal to 0.092 . Naturally, it performs much better than the CAMU array for which $C$ equals 1.2245. Interestingly, performance is in-between those of the 3-sensor UCA (for which $C$ equals 0.1536 ) and the 4-sensor UCA (for which $C$ equals 0.0792 ).

\section{CONCLUSION}

We form an array of two directional sensors and use it to estimate the DOA of a distant coplanar source. Sensors are 


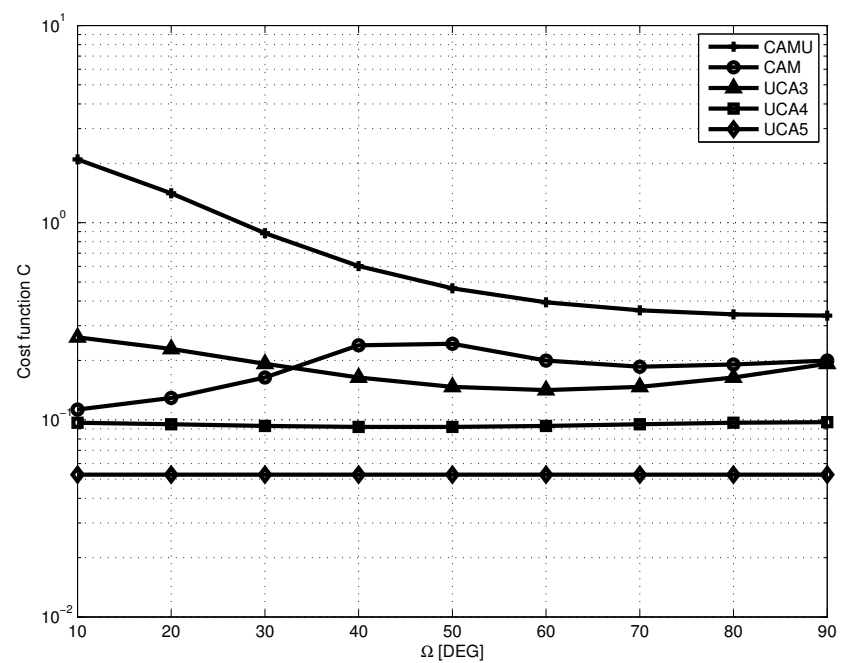

(a)

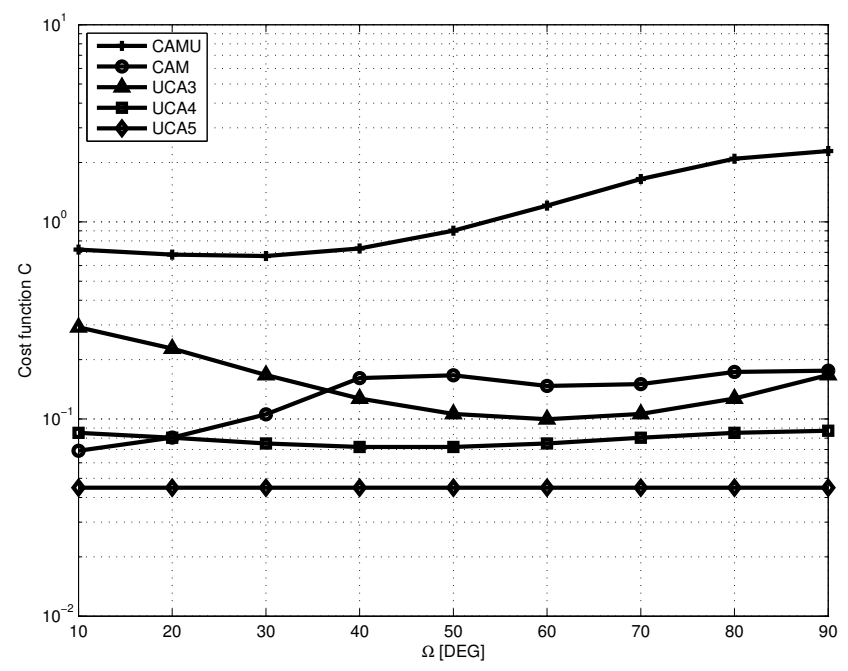

(b)

Fig. 7. Performance of the optimal array, comparatively to the nonadaptive CAMU and UCA arrays for a source PDF characterized by two look directions $\pm \Omega$, with $\Omega=10,20, \ldots, 90$ [DEG]. Sensors are such that $\beta=0.4$ in (a) and $\beta=0.8$ in (b).

positioned and oriented in order to take benefit from the $a$ priori information about the DOA angle and, subsequently, reduce the estimation error. If no a priori is available, a by-default (CAMU) geometry has the advantage of having a finite precision in every direction. If some (normal) a priori is available, the optimal array geometry (calculated off-line) delivers an accuracy comparable to that of a 3 or 4 sensor UCA.

\section{APPENDIX}

\section{A. Proof of (5)}

Derivation of the ARV, as expressed in (4), leads to $\mathbf{a}^{\prime}(\theta)=\left[g_{1}^{\prime}(\theta), g_{2}^{\prime}(\theta) \exp [2 j \pi \rho \cos (\theta-\phi)]-\right.$ $\left.g_{2}(\theta) 2 j \pi \rho \sin (\theta-\phi) \exp [2 j \pi \rho \cos (\theta-\phi)]\right]^{T}, \quad$ so $\quad$ that we obtain $\|\mathbf{a}(\theta)\|^{2}=g_{1}^{2}(\theta)+g_{2}^{2}(\theta)$ and $\left\|\mathbf{a}^{\prime}(\theta)\right\|^{2}=\left[g_{1}^{\prime}(\theta)\right]^{2}+\left[g_{2}^{\prime}(\theta)\right]^{2}+g_{2}^{2}(\theta) 4 \pi^{2} \rho^{2} \sin ^{2}(\theta-\phi)$. Also, $\mathbf{a}^{\prime H}(\theta) \mathbf{a}(\theta)=g_{1}(\theta) g_{1}^{\prime}(\theta)+g_{2}(\theta) g_{2}^{\prime}(\theta)+$ $2 j \pi \rho g_{2}^{2}(\theta) \sin (\theta-\phi) \quad$ results into $\left|\mathbf{a}^{\prime H}(\theta) \mathbf{a}(\theta)\right|^{2}=$ $g_{1}^{2}(\theta)\left[g_{1}^{\prime}(\theta)\right]^{2}+g_{2}^{2}(\theta)\left[g_{2}^{\prime}(\theta)\right]^{2}+2 g_{1}(\theta) g_{1}^{\prime}(\theta) g_{2}(\theta) g_{2}^{\prime}(\theta)+$ $4 \pi^{2} \rho^{2} g_{2}^{4}(\theta) \sin ^{2}(\theta-\phi)$. After substitution into (3), we update $\|\mathbf{a}(\theta)\|^{2} F(\theta)$ as follows

$$
\begin{aligned}
& {\left[g_{1}^{2}(\theta)+g_{2}^{2}(\theta)\right] F(\theta) } \\
= & g_{1}^{2}(\theta)\left[g_{1}^{\prime}(\theta)\right]^{2}+g_{1}^{2}(\theta)\left[g_{2}^{\prime}(\theta)\right]^{2} \\
& +g_{1}^{2}(\theta) g_{2}^{2}(\theta) 4 \pi^{2} \rho^{2} \sin ^{2}(\theta-\phi) \\
& +g_{2}^{2}(\theta)\left[g_{1}^{\prime}(\theta)\right]^{2}+g_{2}^{2}(\theta)\left[g_{2}^{\prime}(\theta)\right]^{2} \\
& +g_{2}^{4}(\theta) 4 \pi^{2} \rho^{2} \sin ^{2}(\theta-\phi) \\
& -g_{1}^{2}(\theta)\left[g_{1}^{\prime}(\theta)\right]^{2}-g_{2}^{2}(\theta)\left[g_{2}^{\prime}(\theta)\right]^{2} \\
& -2 g_{1}(\theta) g_{1}^{\prime}(\theta) g_{2}(\theta) g_{2}^{\prime}(\theta)-4 \pi^{2} \rho^{2} g_{2}^{4}(\theta) \sin ^{2}(\theta-\phi) \\
= & {\left[g_{1}(\theta) g_{2}^{\prime}(\theta)-g_{2}(\theta) g_{1}^{\prime}(\theta)\right]^{2} } \\
& +4 \pi^{2} \rho^{2} g_{1}^{2}(\theta) g_{2}^{2}(\theta) \sin ^{2}(\theta-\phi),
\end{aligned}
$$

which is equivalent to

$$
\begin{aligned}
\frac{g_{1}^{2}(\theta)+g_{2}^{2}(\theta)}{g_{1}^{4}(\theta)} F(\theta)= & \left\{\left[\frac{g_{2}(\theta)}{g_{1}(\theta)}\right]^{\prime}\right\}^{2} \\
& +4 \pi^{2} \rho^{2} \frac{g_{2}^{2}(\theta)}{g_{1}^{2}(\theta)} \sin ^{2}(\theta-\phi)
\end{aligned}
$$

and so to (5).

\section{B. Proof of (10)}

For the considered UCA, the ARV (1) given by $[\mathbf{a}(\theta)]_{m}=$ $g\left(\theta-\phi_{m}\right) \exp \left[j 2 \pi R \lambda \cos \left(\theta-\phi_{m}\right)\right] \hat{=} g_{m} \exp \left(j \tau_{m}\right)$ results in (3) being transformed into

$$
\begin{aligned}
F(\theta) & =\sum_{m=1}^{M} g_{m}^{\prime 2}+\sum_{m=1}^{M} g_{m}^{2} \tau_{m}^{\prime 2} \\
& -\frac{\left(\sum_{m=1}^{M} g_{m} g_{m}^{\prime}\right)^{2}+\left(\sum_{m=1}^{M} g_{m}^{2} \tau_{m}^{\prime}\right)^{2}}{\sum_{m=1}^{M} g_{m}^{2}} .
\end{aligned}
$$

Using property (8), we prove the following identities: $\sum_{m=1}^{M} \sin \left[k\left(\theta-\phi_{m}\right)\right]=0$ for $M>k \geq 1$,

$\sum_{m=1}^{M} \sin \left[k\left(\theta-\phi_{m}\right)\right] \cos \left[l\left(\theta-\phi_{m}\right)\right]=0$ for $M>k+l \geq 2$ and $\sum_{m=1}^{M} \sin \left(\theta-\phi_{m}\right) \cos \left[k\left(\theta-\phi_{m}\right)\right] \cos \left[l\left(\theta-\phi_{m}\right)\right]=0$ for $M>1+k+l \geq 3$. In turn, this allows us to prove, after simple algebraic manipulations, that

$$
\left(\sum_{m=1}^{M} g_{m} g_{m}^{\prime}\right)^{2}+\left(\sum_{m=1}^{M} g_{m}^{2} \tau_{m}^{\prime}\right)^{2}=0 \text { for } M>2 K+1 \text {. }
$$

Now, using the following equalities

$$
\begin{aligned}
\sum_{m=1}^{M} \sin \left[k\left(\theta-\phi_{m}\right)\right] \sin \left[l\left(\theta-\phi_{m}\right)\right] \\
=\sum_{m=1}^{M} \cos \left[k\left(\theta-\phi_{m}\right)\right] \cos \left[l\left(\theta-\phi_{m}\right)\right] \\
= \begin{cases}M / 2 & \text { for } M>k+l \geq 2 \text { and } k=l \\
0 & \text { for } M>k+l \geq 2 \text { and } k \neq l\end{cases}
\end{aligned}
$$




$$
\begin{aligned}
& \sum_{m=1}^{M} \cos \left[2\left(\theta-\phi_{m}\right)\right] \cos \left[k\left(\theta-\phi_{m}\right)\right] \\
& \quad= \begin{cases}M / 2 & \text { for } M>k+2 \geq 3 \text { and } k=2 \\
0 & \text { for } M>k+2 \geq 3 \text { and } k \neq 2\end{cases}
\end{aligned}
$$

$$
\begin{aligned}
& \sum_{m=1}^{M} \cos \left[2\left(\theta-\phi_{m}\right)\right] \cos \left[k\left(\theta-\phi_{m}\right)\right] \cos \left[l\left(\theta-\phi_{m}\right)\right]= \\
& \begin{cases}M / 4 & \text { for } M>k+l+2 \geq 4 \text { and } k=l=1 \\
M / 4 & \text { for } M>k+l+2 \geq 4 \text { and }|k-l|=2 \\
0 & \text { for } M>k+l+2 \geq 4,|k-l| \neq 2, \\
& \quad l \neq 1 \text { and } k \neq 1\end{cases}
\end{aligned}
$$

we can reach the final result in (10).

\section{Proofs of (11)}

First, $\max _{\theta} g_{0}^{2}(1+\cos (\theta))^{2 K}=2^{2 K} g_{0}^{2}$. By applying the binomial equality twice to $(1+\cos (\theta))^{2 K}=\frac{1}{2^{2 K}}\left[2+\left(e^{j \theta}+\right.\right.$ $\left.\left.e^{-j \theta}\right)\right]^{2 K}$, we obtain:

$$
(1+\cos (\theta))^{2 K}=\frac{1}{2^{2 K}}\left[\sum_{k=0}^{2 K} \sum_{\ell=0}^{k} 2^{2 K-k}\left(\begin{array}{c}
2 K \\
k
\end{array}\right)\left(\begin{array}{l}
k \\
\ell
\end{array}\right) e^{j(2 l-k) \theta}\right] .
$$

Using the Euler relationship (8),

$$
\frac{1}{2 \pi} \int_{0}^{2 \pi}(1+\cos (\theta))^{2 K} d \theta=\frac{1}{2^{2 K}} \sum_{n=0}^{K} 2^{2(K-n)}\left(\begin{array}{c}
2 K \\
2 n
\end{array}\right)\left(\begin{array}{c}
2 n \\
n
\end{array}\right)
$$

with $\left(\begin{array}{l}a \\ b\end{array}\right) \hat{=} \frac{a !}{b !(a-b) !}$ concludes the proof.

\section{Proofs of (13)-(16)}

By extensive use of (8), we can prove, after tedious manipulations, that

$$
\begin{aligned}
& \frac{\|\mathbf{a}(\theta)\|^{2}}{M g_{0}^{2}}=1+\beta^{2} \frac{1+\delta_{M, 2} \cos (2 \theta)}{2}, \\
&-\frac{\mathbf{a}^{H}(\theta) \mathbf{a}^{\prime}(\theta)}{g_{0}^{2}}=0, M>3 \\
&=j \frac{3}{2} \pi R \beta^{2} \sin (3 \theta), M=3 \\
&=\beta(\beta+4 j \pi R) \sin (2 \theta), M=2 \\
& \frac{\left\|\mathbf{a}^{\prime}(\theta)\right\|^{2}}{M g_{0}^{2}}=\frac{\beta^{2}}{2}+\pi^{2} R^{2}\left(2+\frac{\beta^{2}}{2}\right), M \geq 5 \\
&=\frac{\beta^{2}}{2}+\pi^{2} R^{2}\left[2+\beta^{2} \sin ^{2}(2 \theta)\right], M=4 \\
&=\frac{\beta^{2}}{2}+\pi^{2} R^{2}\left[2+\frac{\beta^{2}}{2}-2 \beta \cos (3 \theta)\right], M=3 \\
&=\beta^{2} \sin ^{2}(\theta)+\pi^{2} R^{2}\left(4+\beta^{2}\right) \sin ^{2}(2 \theta), M=2
\end{aligned}
$$

where $\delta_{i j}=1$ if $i=j, 0$ otherwise. The above can be used to calculate the exact CRB of the UCA, as expressed by (2) and (3), leading to expressions (13)-(16).

\section{REFERENCES}

[1] Krim, H. and Viberg, M. "Two decades of array signal processing research," IEEE Signal Process. Mag., vol. 13, no. 4, pp. 67-94, Jul. 1996.

[2] Lange, O. and Yang, B. "Optimization of array geometry for directionof-arrival estimation using a priori information," Adv. Radio Sci., vol. 8, pp. 87-94, 2010.

[3] Gazzah, H. "Optimum antenna arrays for isotropic direction finding," IEEE Trans. Aerosp. Electron. Syst., vol. 47, no. 2, pp. 1482-1489, April 2011.

[4] Gazzah, H. and Delmas, J. P. "Direction finding antenna arrays for the randomly located source," IEEE Trans. Signal Process., vol. 60, no. 11, pp. 6063-6068, Nov. 2012.

[5] Gazzah, H. and Delmas, J. P. "CRB based-design of linear antenna arrays for near-field source localization," IEEE Trans. Antennas Propag., vol. 62, no. 4, pp. 1965-1974, April 2014.

[6] Schmidt, R. O. "Multiple emitter location and signal parameter estimation," IEEE Trans. Antennas Propagat., vol. AP-34, no. 3, pp. 276-280, Mar. 1986.

[7] Gazzah, H. and Delmas, J. P. "Spectral efficiency of beamforming-based parameter estimation in the single source case," in Proc. Statistical Signal Processing Workshop (SSP), 2011, pp. 153-156.

[8] Oktel Ü. and Moses, R. L. "A Bayesian approach to array geometry design," IEEE Trans. Signal Process., vol. 53, no. 5, pp. 1919-1923, May 2005.

[9] Bevelacqua, P. J. and Balanis, C. A. "Optimizing antenna array geometry for interference suppression," IEEE Trans. Antennas and Propag., vol. 55, no. 3, pp. 637-641, Mar. 2007.

[10] Schmidt, H., Bellingham, J. G. and Bales, J. W. "Mobile underwater arrays," U.S. Patents No. 5,894,450 (April 1999). https://encrypted.google.com/patents/US5894450

[11] Wang, D., Lermusiaux, P. F. J., Haley, P. J., Eickstedt, D., Leslie, W. G., Schmidt, H. "Acoustically focused adaptive sampling and on-board routing for marine rapid environmental assessment," Elsevier Journal of Marine Systems, 78 (2009), pp. S393-S407.

[12] Jackson, B. R., Rajan, S., Liao, B. and Wang, S. "Direction of arrival estimation using directive antennas in uniform circular arrays," IEEE Trans. Antennas and Propag., vol. 63, no. 2, pp. 736-747, Feb. 2015.

[13] Demmel, F. "Practical Aspects of Design and Application of DirectionFinding Systems," in Classical and Modern Direction-of-Arrival Estimation, Tuncer, T. E. and Friedlander, B., Ed. Academic Press, 2009, pp. 53-92.

[14] Liao, B., Tsui, K.-M. and Chan, S.-C. "Frequency invariant uniform concentric circular arrays with directional elements," IEEE Trans. Aerosp. Electron. Syst., vol. 49, no. 2, pp. 871-884, April 2013.

[15] Balanis C. and Ioannides P., Introduction to Smart Antennas, San Rafael, CA, USA: Morgan \& Claypool, 2007.

[16] Porat, B. and Friedlander, B. "Analysis of the asymptotic relative efficiency of the MUSIC algorithm," IEEE Trans. Acoust., Speech, Signal Process., vol. 36, no. 4, pp. 532-544, Apr. 1988.

[17] Kamkar-Parsi, A. H. and Bouchard, M. "Improved noise power spectrum density estimation for binaural hearing aids operating in a diffuse noise field environment," IEEE Trans. Audio, Speech, Language Process., vol. 17, no. 4, pp. 521-533, May. 2009.

[18] Godara, L. C. and Cantoni, A. "Uniqueness and linear independence of steering vectors in array space," J. Acoust. Soc. Amer, vol. 70, no. 2, pp. 467-475, Aug. 1981.

[19] Gavish, M. and Weiss, A. J. "Array geometry for ambiguity resolution in direction finding," IEEE Trans. Antennas and Propag., vol. 44, no. 6, pp. 143-146, Feb. 1991.

[20] Biguesh, M. and Gazor, S. "On proper antenna pattern for a simple source detection and localization system," IEEE Trans. Antennas and Propag., vol. 57, no. 4, pp. 1073-1080, Apr. 2009.

[21] Gazzah H., and Abed-Meraim, K. "Optimum ambiguity-free directional and omni-directional planar antenna arrays for DOA estimation," IEEE Trans. Signal Processing, vol. 57, no. 10, pp. 3942-3253, Oct. 2009.

[22] Del Val, L., Izquierdo, A., Jimenez, M. I., Villacorta, J. J. and Raboso, M. "Analysis of Directive Sensor Influence on Array Beampatterns," in Microwave and Millimeter Wave Technologies Modern UWB antennas and equipment, Mini, I., Ed. InTech, 2010, pp. 229-242.

[23] Ellis, D. D. "Effect of cardiod and limaçon directional sensors on towed array reverbation response," J. Canadian Acoust. Association, vol. 34, no. 3, pp. 102-103, 2006 


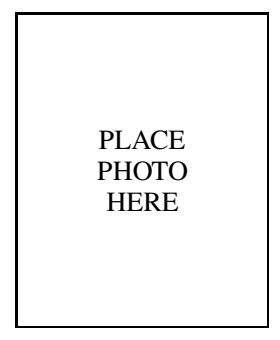

Houcem Gazzah was born in Sousse, Tunisia, in 1971. He received the Engineering degree from the École Supérieure des Communications, Tunis, Tunisia, in 1995, and the M.Sc. and Ph.D. degrees from the École Nationale Supérieure des Télécommunications, Paris, France, in 1997 and 2000 , respectively. He had been a Research Associate with the Centre National de la Recherche Scientifique (LSS/Supélec), Gif-sur-Yvette, France (2001-2003); then with the Institute for Digital Communications, at the University of Edinburgh, UK (2004-2006). He is presently an Assistant Professor with the Department of Electrical and Computer Engineering at the University of Sharjah, UAE. His research interests include antenna array signal processing, (blind) channel identification and equalization, multi-carriers and OFDM systems, adaptive transmission and (bit interleaved) coded modulation.

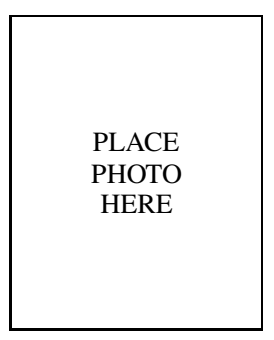

( M'00-SM'06) Jean Pierre Delmas was born in France in 1950. He received the Engineering Degree from École Centrale de Lyon, France in 1973, the Certificat d'Etudes Supérieures from École $\mathrm{Na}$ tionale Supérieure des Télécommunications (ENST), Paris, France in 1982 and the Habilitation à diriger des recherches (HDR) degree from the University of Paris XI, Orsay, France in 2001. Since 1980, he has been with Telecom SudParis (formerly INT) where he is presently a Professor in the CITI department. He was deputy director (2005-2010) and director (2011-2014) of UMR 5157 (CNRS laboratory). His teaching and research interests are in the area of statistical signal processing with application to communication and antenna arrays. Prof. Delmas has served as an Associate Editor for the IEEE Transactions on Signal Processing (2002-2006) and (20102014), and presently for Signal Processing (Elsevier). He is a member of the IEEE Sensor Array and Multichannel (SAM) Technical Committee and a IEEE Senior Member. He is author and co-author of more than 120 papers (journals, conferences and book chapters).

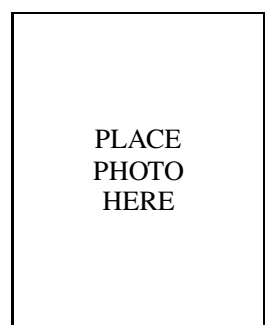

Sérgio M. Jesus received his "Doctorat EsSciences" in Engineering Sciences from the Université de Nice, France in 1986. From 1986 to 1992 he was a staff scientist at the SACLANT Undersea Research Centre, La Spezia, Italy, in the Ambient Noise and Signal Processing Groups. During that period he was involved with underwater acoustic field noise directionality and early studies on target detection using matched field processing. In 1992 he joined the Electrical Engineering and Computer Science Department at the University of Algarve, Faro, Portugal where he is currently full professor. His interests are underwater acoustics array signal processing, model-based inversion, ocean and seafloor tomography and underwater acoustic communications. He has coordinated EU projects UAN and OAEx. He is member of IEEE, EURASIP and of the Acoustical Society of America. 